\title{
10 Der Fragetyp STACK
}

\author{
Miriam Weigel
}

Das Plug-in STACK (System for Teaching and Assessment using a Computer algebra Kernel) wurde ursprünglich 2004 von Chris Sangwin (University of Edinburgh) für das Lernmanagementsystem (LMS) Moodle erstellt. Im Jahr 2013 wurde STACK dann im Auftrag von optes von Fred Neumann und Jesus Copado (FAU Erlangen) für das LMS ILIAS implementiert.

Für die Testteilnehmer*innen sind STACK-Fragen im Allgemeinen kaum von Lückentextfragen zu unterscheiden. Lediglich ein kleines Häkchen an der Eingabelücke, das zur Antwortüberprüfung dient, gibt einen Hinweis darauf, dass es sich um einen anderen Fragetyp handelt (siehe Abbildung 1). In das Eingabefeld einer STACK-Frage können im Unterschied zu numerischen Lückentextfragen nicht nur Zahlenwerte, sondern mittels ASCIIMATH-Syntax auch mathematische Terme und Formeln eingegeben werden (siehe Abbildung 1). Da die Eingabe solcher Terme sehr fehleranfällig ist, können die Teilnehmer*innen mit Hilfe des Häkchen-Buttons die Syntax ihrer Eingabe überprüfen, ohne dass die Antwort bewertet wird. Erst wenn der Button „Rückmeldung anfordern“ ausgewählt wird, wird die Eingabe mit Hilfe des Computeralgebrasystems (CAS) Maxima auf ihre mathematischen Eigenschaften geprüft und abschließend bewertet. Durch diese Form der Antwortüberprüfung werden Fehleranalysen, Schritt-für-Schritt-Anleitungen und offene Fragestellungen mit beliebig vielen Lösungen ermöglicht (Weigel et al. 2018). Diese offenen Frageformate waren bisher in Online-Aufgaben nur sehr schwer oder gar nicht abbildbar.

$\checkmark$ Wichtige Hinweise zur Eingabe-Syntax

Geben Sie eine Funktion $f$ mit den Nullstellen $x_{1}=4, x_{2}=3$ und $x_{3}=-5$ an!

$$
\begin{aligned}
& f(x)=(x-4)^{*}(x-3)^{*}(x-5) \\
& (x-4)^{*}(x-3) *(x-5) \text { wurde wie folgt interpretiert: }(x-4) \cdot(x-3) \cdot(x-5)
\end{aligned}
$$

Rückmeldung anfordern

Abbildung 1: STACK-Aufgabe mit Syntaxüberprüfung 
Für Aufgabenersteller*innen bietet der Fragetyp STACK durch die Anbindung an das CAS Maxima eine Vielzahl an Möglichkeiten.

Neben dem Einsatz von Zufallszahlen in der Aufgabenstellung wird auch die Manipulation der Rückmeldung und somit ein adaptives und aufschlussreiches Feedback ermöglicht (siehe Abbildung 2). Die Rückmeldung (korrekt / teilweise korrekt / nicht korrekt / Lösungsweg etc.) basiert auf der Eingabe des Teilnehmenden und der zufällig erzeugten Variablen. Die Zufallszahlen können auf einen bestimmten Wertebereich eingeschränkt werden, sodass ausschließlich realistische bzw. sinnvolle Zahlenwerte generiert werden. Somit müssen nicht mehrere Aufgaben erstellt werden, um einen bestimmten Aufgabentyp ausreichend darzustellen, sondern es können mit einer STACK-Frage und geschickt gewählten Zufallsvariablen Dutzende Versionen erschaffen werden. Die Testteilnehmenden erhalten so bei jedem Aufruf des Tests eine andere Aufgabenvariante, wodurch der Übungseffekt verstärkt und das Wiederholen des Tests gefördert wird.

Zudem können statische (mit Hilfe von Maxima oder HTML) und dynamische (mit Hilfe von JSX-Graph) Grafiken in den Fragetext oder die Rückmeldung eingebaut werden. Diese Grafiken können sich auf die Zufallswerte beziehen und somit automatisch an diese und die studentische Antwort angepasst werden (siehe Abbildung 2). Dies ermöglicht beispielsweise eine Gegenüberstellung der studentischen Antwort mit der Musterlösung in grafischer Form (Weigel et al. 2019).

Da die Werte einer Aufgabe immer wieder neu berechnet werden, sind STACK-Aufgaben ideal für den Einsatz im Übungsbereich (Sangwin 2013). Viele Studienanfänger*innen kennen die ASCIIMATH-Syntax von ihren grafikfähigen Taschenrechnern. Um jedoch Eingabefehler zu vermeiden, besitzt jede STACKFrage in den optes-Kursen den Reiter „Wichtige Hinweise zur Eingabe-Syntax“ mit Erklärungen zur korrekten Eingabe der ASCIIMATH-Syntax (siehe Abbildung 1). Im optes-Teilprojekt „Formatives E-Assessment und Propädeutika“ wird bei der Entwicklung von Aufgaben für das Selbststudium je nach Lernziel und Anspruchsniveau über den Aufgabentyp entschieden. Zur Auflockerung des Lernprozesses werden verschiedene Aufgabentypen gemischt. In den Trainings zu den Lernmodulen werden beispielsweise Single-Choice-, Lückentext-, Zuordnungsund STACK-Fragen verwendet.

Seit 2014 werden an der DHBW Mannheim betreute E-Learning-Kurse angeboten. Im Sommer 2018 wurde der Einsatz des Fragetyps STACK mit einer kleinen Gruppe von Studienanfänger*innen $(n=19)$ pilotiert, die an fünf Präsenzterminen an der DHBW Mannheim teilnahmen. Aufgrund der positiven Evaluationsergebnisse wurden anschließend 2019 im Mathematikvorkurs der Fakultät Technik der DHBW Mannheim in allen Trainings zu den Lernmodulen des optesKerncurriculums STACK-Fragen aufgenommen (siehe Abbildung 3). Für das Sommersemester 2020 ist der Einsatz von STACK-Fragen auch in Lehrveranstaltungen geplant. 


\section{Ihre Antwort ist teilweise korrekt.}

Ihre Funktion nimmt für $x=-5$ nicht den Wert 0 an.

Das Schaubild zeigt die Musterlösung und Ihre Antwort:

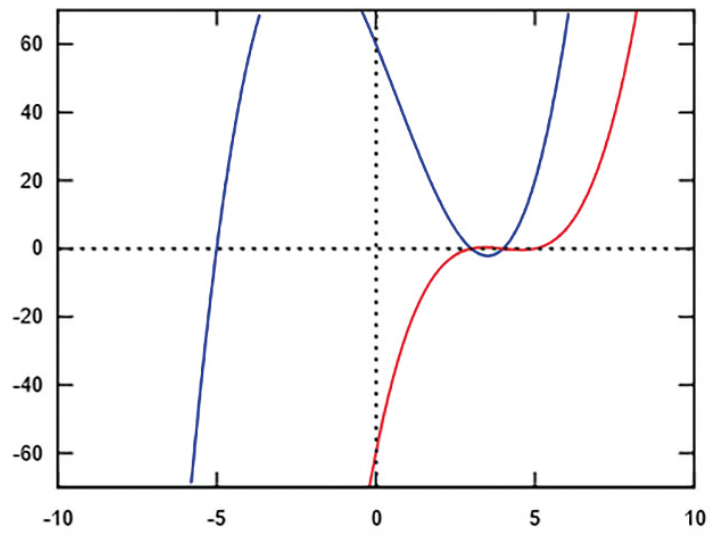

\section{Erläuterung:}

Wir suchen eine Funktion $f(x)$, die die Bedingung $f(4)=f(3)=f(-5)=0$ erfüllt. Eine Funktion mit diesen Eigenschaften ist beispielsweise:

$$
f(x)=(x-4) \cdot(x-3) \cdot(x+5)=x^{3}-2 \cdot x^{2}-23 \cdot x+60
$$

Abbildung 2: Rückmeldung zu der Eingabe aus Abbildung 1

Digitalisiertes Feedback ist zeit- und ortsunabhängig und daher von großem Nutzen für Lernende und Lehrende. Bei der Entscheidung, welcher Aufgabentyp zum Einsatz kommt, sind neben dem Lernziel sowie dem Komplexitäts- und Schwierigkeitsgrad der Aufgabenstellung auch die meta-kognitiven Anforderungen zu berücksichtigen, die durch das technische Handling entstehen können. Die eher unflexiblen Multiple-Choice-Aufgaben sind aufgrund ihrer Niederschwelligkeit bei der Eingabe für unerfahrene E-Learning-Teilnehmer*innen von Vorteil. Jedoch birgt das Aufgabenformat ein hohes „Raterisiko“ (Sangwin 2013). Abhilfe schaffen Aufgabenformate, bei denen ein mathematischer Ausdruck eingegeben werden muss (z. B. Lückentext- oder STACK-Aufgaben). Aufgaben, die die Abgabe ganzer Rechenschritte oder mathematischer Lösungsansätze verlangen, erfordern die individuelle Korrektur und Rückmeldung einer Lehrperson, und sind daher nur im Rahmen betreuter Online-Vorkurse anwendbar. 
Besonders wichtig für die Feedbackgestaltung von Online-Fragen ist es, die Ziele im Lernprozess zu definieren. Steht die Selbstkontrolle im Mittelpunkt, so reichen Frageformate mit einfachem und flachem Feedback aus. Wird ein qualitatives Feedback mit Fehleranalyse benötigt, dann bietet der Fragetyp STACK hervorragende Möglichkeiten für die Gestaltung von automatisiertem und adaptivem Feedback.

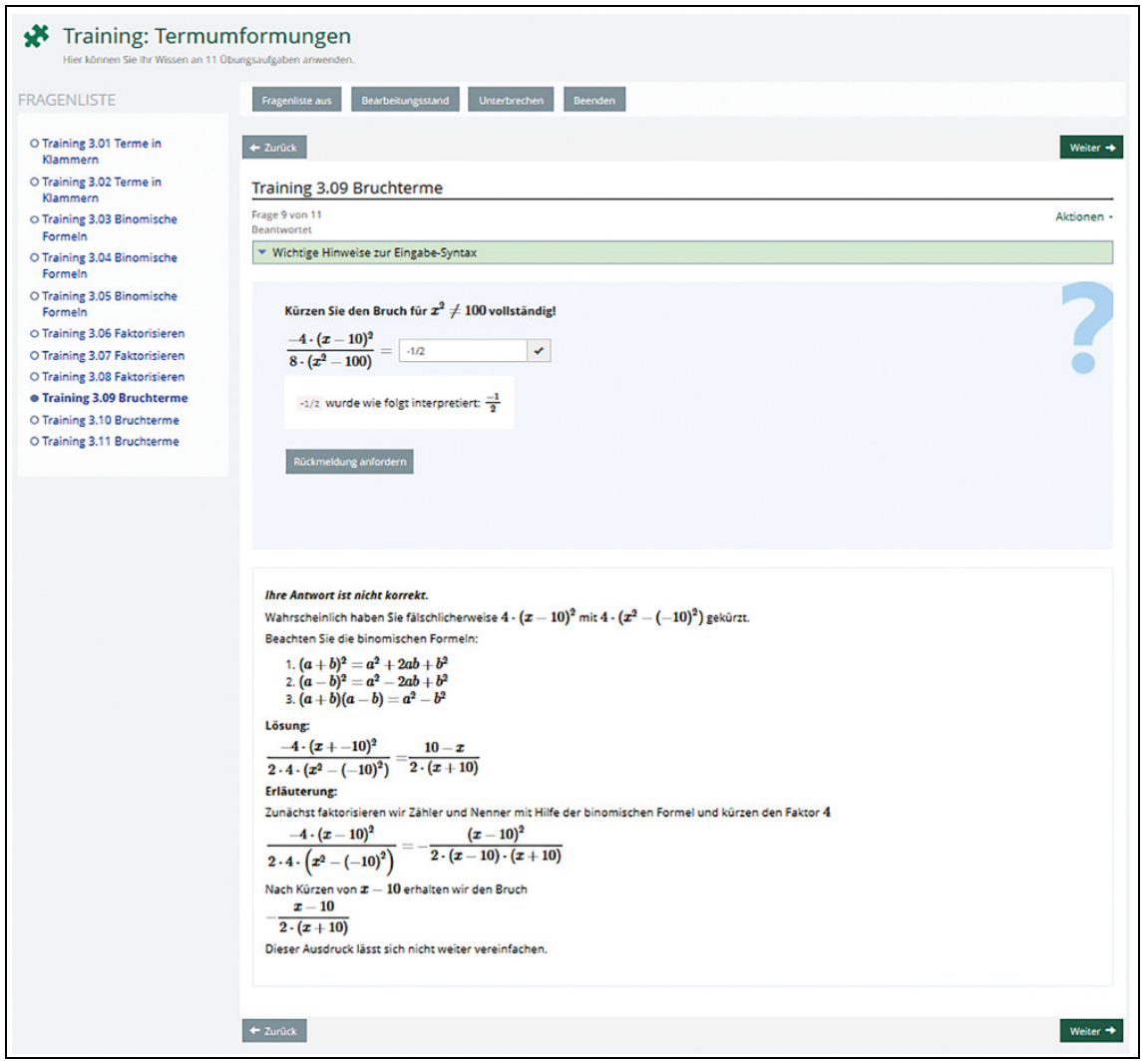

Abbildung 3: STACK-Aufgabe im Training Termumformungen (Kurs Arithmetik) 


\section{Literatur}

Sangwin, C. (2013). Computer Aided Assessment of Mathematics Using Stack. Oxford: Oxford University Press.

Weigel, M., Hübl, R., Podgayetskaya, T. \& Derr, K. (2018). Potential von STACK-Aufgaben im formativen eAssessment: Automatisiertes Feedback und Fehleranalyse. In Fachgruppe Didaktik der Mathematik der Universität Paderborn (Hrsg.), Beiträge zum Mathematikunterricht (S. 1419-1422). Münster: WTM.

Weigel, M., Derr, K., Hübl, R. \& Podgayetskaya, T. (2019). STACK-Aufgaben im formativen eAssessment: Einsatzmöglichkeiten des Feedbacks. In Contributions to the 1st International STACK conference 2018. Friedrich-Alexander-Universität, Nürnberg, Germany.

Open Access Dieses Kapitel wird unter der Creative Commons Namensnennung 4.0 International Lizenz (http://creativecommons.org/licenses/by/4.0/deed. de) veröffentlicht, welche die Nutzung, Vervielfältigung, Bearbeitung, Verbreitung und Wiedergabe in jeglichem Medium und Format erlaubt, sofern Sie den/ die ursprünglichen Autor(en) und die Quelle ordnungsgemäß nennen, einen Link zur Creative Commons Lizenz beifügen und angeben, ob Änderungen vorgenommen wurden.

Die in diesem Kapitel enthaltenen Bilder und sonstiges Drittmaterial unterliegen ebenfalls der genannten Creative Commons Lizenz, sofern sich aus der Abbildungslegende nichts anderes ergibt. Sofern das betreffende Material nicht unter der genannten Creative Commons Lizenz steht und die betreffende Handlung nicht nach gesetzlichen Vorschriften erlaubt ist, ist für die oben aufgeführten Weiterverwendungen des Materials die Einwilligung des jeweiligen Rechteinhabers einzuholen.

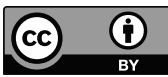

